\title{
Early postnatal methoxychlor exposure inhibits folliculogenesis and stimulates anti-Mullerian hormone production in the rat ovary
}

\author{
Mehmet Uzumcu, Peter E Kuhn, Jason E Marano, AnnMarie E Armenti and Lisa Passantino \\ Department of Animal Sciences, School of Environmental and Biological Sciences, Rutgers, The State University of New Jersey, 84 Lipman Drive, New \\ Brunswick, New Jersey 08901-8525, USA \\ (Requests for offprints should be addressed to M Uzumcu; Email: uzumcu@aesop.rutgers.edu)
}

\begin{abstract}
Methoxychlor [1,1,1-trichloro-2,2-bis(4-methoxyphenyl) ethane; MXC] is a chlorinated hydrocarbon pesticide commonly used in the United States as a replacement for DDT [1,1,1-trichloro-2,2-bis(p-chlorophenyl)ethane]. While MXC is a weak estrogenic compound, its more active, major metabolite [2,2-bis( $p$-hydroxyphenyl)-1,1,1-trichloroethane; HPTE] shows estrogenic, anti-estrogenic, or anti-androgenic properties depending on the receptor subtype with which it interacts. Anti-Mullerian hormone (AMH) is a paracrine factor that suppresses initial follicle recruitment in the ovary. Studies have shown the effects of exposure to MXC on adult ovarian morphology and function. However, the effect of exposure to MXC at an early postnatal stage on pre-pubertal follicular development and ovarian AMH production has not been studied. Around postnatal day (P) 4, most of the primordial follicular assembly in rats is complete, and a large number of primordial follicles transition into the primary follicle stage, a process that is inhibited by estrogen. The objective of this study was to examine the effect of early postnatal (P3-P10) MXC exposure on ovarian morphology and size, follicle number, and AMH production in the pre-pubertal (P20) rat ovary and to investigate the effect of HPTE on AMH production in immature rat granulosa cells in vitro. Female rats were injected (s.c.) daily with vehicle (control) or 1, 10, 50, 100, or $500 \mathrm{mg}$ $\mathrm{MXC} / \mathrm{kg}$ per day (referred to here as $1 \mathrm{MXC}, 10 \mathrm{MXC}$, and so forth.) between P3 and P10. On P20, uterine and ovarian weights were determined, ovarian histology was examined, and follicles were counted and classified into primordial, primary, secondary, pre-antral, or antral stages using the two largest serial sections at the center of the ovary. Ovarian AMH production

was examined using immunohistochemistry and western blot analysis. The effect of HPTE $(0 \cdot 5-25 \mu \mathrm{M})$ on AMH production in cultured immature rat granulosa cells was determined by western blot analysis. Ovarian weight was reduced by 50, 100, and 500MXC $(P<0 \cdot 01)$. MXC treatment inhibited folliculogenesis. Both 100 and 500MXC had a reduced number of antral follicles $(P<0 \cdot 05)$ with a concomitant increase in pre-antral follicles $(P<0 \cdot 05)$. Follicle numbers were not significantly affected by 1,10 , or 50MXC. Total follicle number and the number of primordial, primary, or secondary stage follicles were not significantly different in all treatment groups. Immunohistochemistry showed that MXC-treated ovaries had more $\mathrm{AMH}$-positive follicles with stronger $\mathrm{AMH}$ immunostaining. Western blot analysis showed that AMH production was $1.6 \pm$ $0 \cdot 2,1 \cdot 85 \pm 0 \cdot 6$, and $2 \cdot 2 \pm 0 \cdot 5$ times higher in the 50,100 , and $500 \mathrm{MXC}$ ovaries as compared with the control ovaries respectively $(P<0 \cdot 05)$. Granulosa cells treated with 1 or $5 \mu \mathrm{M}$ HPTE had significantly greater AMH production $(P<0 \cdot 05)$. These results demonstrate that $\mathrm{MXC}$ inhibits early ovarian development and stimulates AMH production directly in the rat ovary. In addition, HPTE was shown to stimulate AMH production in rat granulosa cells. Endocrine disruptors are widespread in the environment, and MXC represents a model endocrine disruptor due to the multiple actions of its metabolites. This study confirms that the endocrine disruptor MXC inhibits follicular development and demonstrates for the first time that MXC and HPTE directly stimulate $\mathrm{AMH}$ production in the ovary. This novel finding suggests that elevated AMH may play a role in MXC's inhibitory effect in the ovary. Journal of Endocrinology (2006) 191, 549-558
\end{abstract}

\section{Introduction}

The primary functions of the mammalian ovary are steroidogenesis and ovulation, processes that are intricately connected to folliculogenesis. Primordial follicles are formed from oocyte nests after synchronous oocyte apoptosis occurs during the perinatal period in mice (Pepling \& Spradling 2001). Each primordial follicle consists of an oocyte surrounded by squamous, non-growing pre-granulosa cells.
The formation is completed by around postnatal day (P) 3 or 4 in rats (Kezele \& Skinner 2003). Most primordial follicles remain quiescent, but some grow and transition into the primary follicle stage. Each primary follicle consists of an oocyte surrounded by a single layer of proliferating cuboidal granulosa cells (Hirshfield 1991).

Early follicular development is primarily controlled by paracrine growth factors. Some growth factors, such as kit ligand and growth differentiation factor-9 stimulate follicular 
development, and their dysfunction or absence results in deficiencies in this process (Huang et al. 1993, Bedell et al. 1995, Dong et al. 1996). Other growth factors, including antiMullerian hormone (AMH), inhibit early folliculogenesis. In general, $\mathrm{AMH}$ is not expressed in primordial follicles, but only expressed in the primary follicle through small antral follicle stages. In pre-ovulatory, large antral follicles, $\mathrm{AMH}$ expression disappears or is very limited. AMH-expressing follicles appear to inhibit initial recruitment of primordial follicles, since increased initial follicular recruitment occurs in AMH-deficient mice (Durlinger et al. 1999). In addition, cultured neonatal mice ovaries treated with $\mathrm{AMH}$ have fewer growing follicles as compared with untreated ovaries (Durlinger et al. 2002). Furthermore, AMH may also inhibit gonadotropin-stimulated cyclic follicle recruitment, since AMH inhibits follicle-stimulating hormone (FSH)-stimulated aromatase activity and luteinizing hormone $(\mathrm{LH})$ receptor expression in granulosa cells (di Clemente et al. 1994), thereby attenuating the effects of FSH on follicular development in the ovary. Thus, AMH appears to be a physiological brake in the ovary.

Neonatal estrogen treatment inhibits folliculogenesis (Ikeda et al. 2001) and stimulates $\mathrm{AMH}$ expression in the ovary (Ikeda et al. 2002). A recent study showed that estradiol treatment inhibits the primordial-to-primary follicle transition in neonatal ovaries in vitro, suggesting a direct action of the steroid on the ovary (Kezele \& Skinner 2003). Methoxychlor [1,1,1-trichloro-2,2-bis(4-methoxyphenyl) ethane; MXC] is an organochlorine pesticide used as a replacement for DDT [1,1,1-trichloro-2,2-bis(p-chlorophenyl)ethane]. MXC is estrogenic in vivo and has low affinity for the estrogen receptor (Hall et al. 1997). One of the primary metabolites of $\mathrm{MXC}$ is 2,2-bis( $p$-hydroxyphenyl)1,1,1-trichloroethane (HPTE). HPTE shows estrogenic, anti-estrogenic, or anti-androgenic activities depending on the receptor subtype (Maness et al. 1998, Gaido et al. 2000). MXC is considered to be a model endocrine disruptor (Cummings 1997) with direct effects on the antral follicle (Miller et al. 2005).

Studies have shown the effect of relatively long-term MXC exposure on adult ovarian morphology and female fertility. Exposure to $\mathrm{MXC}$ during the first 14 days of life leads to irregular estrous cycles and causes the formation of either follicular cysts or ovarian atrophy in adult mice depending on the dose (Eroschenko et al. 1995). Rats were exposed to $5-150 \mathrm{mg} / \mathrm{kg}$ per day MXC 1 week before and 6 weeks after birth, and reproductive parameters were examined (Chapin et al. 1997). Doses of 50 and $100 \mathrm{mg} / \mathrm{kg}$ per day MXC stimulated early vaginal opening, inhibited ovarian development, caused irregular reproductive cycles, and reduced fecundity in rats. Exposure to $32 \mathrm{mg} / \mathrm{kg} \mathrm{MXC}$ for 20 days reduces antral follicle number by atresia in mice (Borgeest et al. 2002). However, the effect of brief neonatal (P3-P10) exposure to MXC on pre-pubertal (P20) ovarian folliculogenesis and $\mathrm{AMH}$ production in the ovary has not been studied.
The objective of this study was to examine the effect of the estrogenic endocrine disruptor MXC on the rat ovary when administered during the primordial-to-primary follicle transition (P3-P10), which Kezele \& Skinner (2003) demonstrated to be inhibited by estradiol-17 $\beta$. Tissues were collected for analysis at a pre-pubertal age (P20) before cyclic gonadotropin secretion starts in order to assess the effects of MXC on early folliculogenesis and AMH expression in the ovary. In the present study, as an exposure period P3-P10 was selected, since the formation of primordial follicles is mostly completed around P3 or P4 (Kezele \& Skinner 2003). While most primordial follicles remain quiescent, a relatively large number of newly formed primordial follicles undergo the primordial-to-primary follicle transition right after follicle formation (McGee \& Hsueh 2000). After the transition of primordial follicles into the primary follicle stage, they are destined for either ovulation or atresia (Hirshfield 1991). Both of these processes deplete the limited number of follicles, ending the reproductive lifespan of a female.

\section{Materials and Methods}

\section{Animals}

Neonatal female Sprague-Dawley rats were injected daily (s.c.) with $1,10,50,100$, or $500 \mathrm{mg} / \mathrm{kg}$ per day methoxychlor between $\mathrm{P} 3$ and $\mathrm{P} 10$ ( $\mathrm{P} 0=$ day of birth). Control rats were injected with vehicle, $25 \mu$ DMSO:sesame oil (1:2). The s.c. injection route was used for a more precise dosing.

To facilitate reading, the $1 \mathrm{mg} / \mathrm{kg}$ per day MXC group will be referred to as ' $1 \mathrm{MXC}$ ', the $10 \mathrm{mg} / \mathrm{kg}$ per day MXC group will be referred to as '10MXC', and so forth (Chapin et al. 1997). The MXC dose was calculated according to the body weight (BW) on the first day of the injections. The average BWs were not different between the groups and ranged from $9 \cdot 4$ to $9 \cdot 6 \mathrm{~g}$ on P3. The doses that were used in this study are comparable with the doses that have been used in previous studies (5-800 mg/kg per day; Chapin et al. 1997, Hall et al. 1997). Environmental levels of MXC range from 40 to 160 ppm (mg/l) in waters downstream of MXC-sprayed areas (cited in Miller et al. 2005), which is within the range of doses in the present study. Since the actual MXC intake by humans averages only $100 \mathrm{ng} /$ day (Gunderson 1988), MXC is not a specific concern for human health. However, many endocrine disruptors show estrogenic, anti-estrogenic, or anti-androgenic properties. Since MXC's major metabolite, HPTE, has estrogenic, anti-estrogenic, and anti-androgenic actions, MXC was selected as a prototype endocrine disruptor in this study.

The animal care followed the Rutgers University Animal Care and Facilities Committee's guidelines. On P20, the rats were euthanized by $\mathrm{CO}_{2}$ inhalation and the uteri and ovaries were collected. After organ weights were determined, one ovary was used for histology, follicle counting, and $\mathrm{AMH}$ immunohistochemistry (IHC) while the other was used for western blot analysis. 


\section{Ovarian histology}

Ovaries were fixed in Bouin's fixative for $2 \mathrm{~h}$ at room temperature and stored in $70 \%$ ethanol at $4{ }^{\circ} \mathrm{C}$ until embedding in paraffin. Paraffin-embedded tissues were sectioned at 4-5 $\mu \mathrm{M}$. One of the sections from the largest cross section at the center of the ovary was used for hematoxylin and eosin ( $\mathrm{H}$ and $\mathrm{E}$ ) staining and was imaged using light microscopy to determine size of the ovary and follicle numbers and stages in the ovary.

\section{Follicle numbers and stages}

Using two sections from the largest cross section of the ovary, the number of follicles in each developmental stage was counted (Parrott \& Skinner 1999). The follicles was classified according to previously published studies (Oktay et al. 1995). Primordial: an oocyte surrounded by squamous pre-granulosa cells of which not more than one is an enlarged granulosa cell. Primary: a relatively larger oocyte surrounded by at least two or more cuboidal granulosa cells. At the early primary follicle stage, some of the granulosa cells may still be squamous. Secondary (transitional): an oocyte surrounded by two to three layers of cuboidal granulosa cells. Pre-antral: an oocyte surrounded by more than three layers of granulosa cells with no apparent antrum. Antral: an oocyte surrounded by multiple layers of granulosa cells with an antrum. Unknown follicles: granulosa cells surrounded by a theca layer without a clear oocyte present.

\section{AMH immunohistochemistry}

One of the sections was used for IHC as previously described (Uzumcu et al. 2000). The sections were deparaffinized, rehydrated, and microwaved in $0 \cdot 01 \mathrm{M}$ citrate buffer $(\mathrm{pH} 6 \cdot 0$ ) for antigen retrieval. The sections were then blocked with $1 \cdot 5 \%$ normal serum for $10 \mathrm{~min}$ at room temperature. A polyclonal goat anti-AMH antibody (sc-6886, Santa Cruz Biotechnology, Santa Cruz, CA, USA) was used as the primary antibody. The sections were incubated with the primary antibody (1:200) overnight at room temperature. The negative control sections, instead of the primary antibody, were incubated with normal goat $\operatorname{IgG}$ or with primary antibody plus excess $(10 \times)$ blocking peptide. Immunoreactivities were visualized using the ABC Staining System (Santa Cruz Biotechnology) following the manufacturer's instructions. Sections were counterstained with Mayer's hematoxylin.

\section{Granulosa cell culture}

The granulosa cells were prepared as described previously with some modification (Uzumcu \& Lin 1994, Zachow \& Uzumcu 2006). Briefly, to increase cell harvest, immature female rats (21-23 days old) were injected (s.c.) with $250 \mu \mathrm{g}$ diethylstilbestrol (DES) in $100 \mu$ l sesame oil daily for 5 days.
Eight hours after the last DES injection, the rats were killed. Ovaries were removed from the animal, isolated from the associated fat, oviduct, and bursa ovary, and then placed in ice-cold Dulbecco's modified Eagle's medium: Ham's nutrient mixture F-12 (DMEM/F-12). Granulosa cells were isolated using a non-enzymatic needle puncture method with a sterile bundle of beading needles. Following two washes, the cells were plated at a density of approximately $4 \times 10^{5}$ viable cells $/ \mathrm{ml}$ in a culture containing $10 \mathrm{ml}$ DMEM/F-12 medium supplemented with 5\% FBS, $100 \mathrm{U} / \mathrm{ml}$ penicillin, and $100 \mathrm{~g} /$ $\mathrm{ml}$ streptomycin sulfate for $24 \mathrm{~h}$. The cells were treated with HPTE $(0 \cdot 5,1,5,10$, or $25 \mu \mathrm{M})$ in $5 \mathrm{ml}$ serum-free DMEM/ F-12 for $24 \mathrm{~h}$. The cells were harvested for AMH western blot analysis at the end of treatment. The experiment was repeated with granulosa cells harvested from immature rats that were injected (s.c.) with $15 \mathrm{IU}$ equine chorionic gonadotropin (eCG).

\section{Western blot analysis}

Western blot analysis was performed as previously described (Uzumcu et al. 2002). The ovaries were lysed with $1 \times$ cell lysis buffer (Cell Signaling Technology, Beverly, MA, USA). The protein concentration in the supernatants was estimated using the DC Protein Assay Kit II (Bio-Rad). Approximately, $60 \mu \mathrm{g}$ protein in the sample-loading buffer were boiled for $5 \mathrm{~min}$ and electrophoresed on a $10-12 \%$ SDS gel. The protein was subsequently transferred onto a nitrocellulose membrane and probed with specific antibodies for $\mathrm{AMH}$ and actin (AMH, sc-6886; actin, sc-1616; Santa Cruz Biotechnology). Following incubation with a secondary antibody, the specific antigen-antibody complex was visualized using ECL Advance Western Blotting Detection Kit (Amersham Biosciences) and viewed by autoradiography. The relative intensity was determined using actin as the loading control.

\section{Statistical analysis}

Organ weight, follicle numbers, and western blot data were analyzed using GraphPad Prism (GraphPad Software, San Diego, CA, USA). The values were expressed as the mean \pm S.E.M. Statistical analysis was performed and the difference between the means of treatments and the respective controls was determined using one-way ANOVA followed by Dunnett's multiple comparison test. For the ovarian weight, histology, IHC, follicle number, and whole-ovary $\mathrm{AMH}$ western blot analysis, experiments were repeated two to five times, using at least two to three animals for each treatment in each experimental repeat. Therefore, 5-11 animals were used for each treatment group. The granulosa cell culture $\mathrm{AMH}$ western blot analysis experiment was repeated thrice using one replicate (i.e. one culture flask) for each treatment in one experiment. For each experiment, the cells were obtained from seven to eight animals and combined. A statistically significant difference was confirmed at $P<0 \cdot 05$. 


\section{Results}

\section{Ovarian weights}

Daily MXC treatment between P3 and P10 decreased ovarian weight and increased uterine weight by P20 (Fig. 1). The decrease in ovarian weight was significant for 50,100, and 500MXC $(P<0 \cdot 01)$. Only 100 and 500MXC caused a significant increase in uterine weight $(P<0 \cdot 01)$. None of the MXC doses caused systemic toxicological effects as indicated by general appearance, and no significant change in body weight occurred. On P20, the average BWs for all the groups ranged between 51.0 and $52.5 \mathrm{~g}(P>0.05)$. In a separate experiment, the body weight of rats that were treated daily with MXC (1-500 $\mathrm{mg} / \mathrm{kg}$ per day) did not significantly change during the treatments (data not shown).

\section{Ovarian histology}

Daily MXC treatment between P3 and P10 caused a reduction in the size of the ovary and inhibited folliculogenesis (Fig. 2). The 50 and $500 \mathrm{MXC}$ treatments reduced ovarian size by approximately 35 and $80 \%$ respectively (Fig. 2C and E), as compared with the controls (Fig. 2A). The size reduction by 100MXC was approximately $75 \%$ (not shown). While control ovaries had many small, medium, and large antral follicles (Fig. 2B), there was a dose-dependent reduction in the number of antral follicles in the ovaries of 50MXC (Fig. 2D), 100MXC (not shown), and 500MXC (Fig. 2F). The size, histology, and follicle composition of 1 and 10MXC were similar to those of the controls (not shown). The 50MXC dose eliminated most of the large antral follicles, but some small and medium-sized antral follicles remained (Fig. 2D). Ovaries of 500MXC had few or no antral follicles (Fig. 2F). The 100MXC effect was similar to that of 500MXC (data not shown).

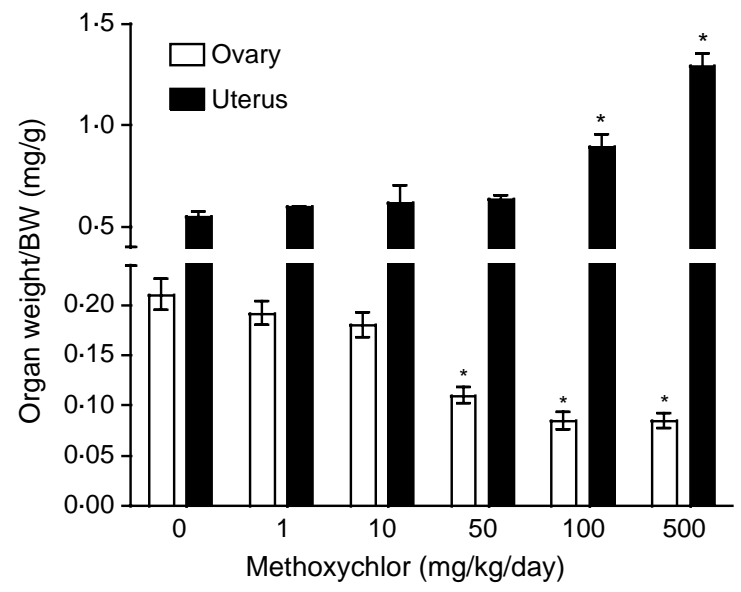

Figure 1 Effect of early postnatal methoxychlor (MXC) exposure on P20 rat ovarian and uterine weights. The procedures were carried out as described in Materials and Methods. Daily MXC injections (s.c.) between $\mathrm{P} 3$ and $\mathrm{P} 10$ reduced ovarian weight and increased uterine weight relative to body weight (BW) by P20 $(n=5-11) .{ }^{*} P<0 \cdot 01$.
Follicle numbers and stages

In order to determine more precisely any effects of different doses of MXC on follicle numbers and stages, follicles in all stages of development were counted in two serial sections from the largest cross-sectional area at the center of the ovary (Fig. 3). On average, a section in control ovaries had 99.28 \pm $8 \cdot 0$ total follicles that were distributed among primordial follicles, $9 \cdot 0 \pm 2 \cdot 0$; primary follicles, $22 \cdot 9 \pm 4 \cdot 2$; transitional (secondary) follicles, $4 \cdot 1 \pm 1 \cdot 9$; pre-antral follicles, $7 \cdot 7 \pm 3 \cdot 3$; and antral follicles, $12 \cdot 3 \pm 1 \cdot 5$. The total number offollicles was unaffected by any of the treatments. Both 100 and 500MXC reduced the antral follicle number significantly with a concomitant increase in the pre-antral follicle stage $(P<0 \cdot 05)$. The number of follicles in primordial, primary, and transitional stages did not differ among all treatment groups $(P>0 \cdot 05)$, nor did the number of unknown follicles differ between treatment groups $(P>0 \cdot 05)$.

\section{AMH immunohistochemistry}

Immunohistochemistry showed that AMH was produced by granulosa cells of primary, secondary, pre-antral, and small antral follicles (Fig. 4). In medium and large antral follicles, AMH immunostaining was reduced. This limited AMH production was primarily observed in cumulus granulosa cells (Fig. 4A; arrowheads). MXC-treated ovaries had many more AMH-positive follicles with stronger AMH immunostaining (Fig. 4C, E and G) as compared with the control ovaries (Fig. 4A). In 1 and 10MXC ovaries, the number of $\mathrm{AMH}$-positive follicles and the intensity of the staining was similar to the control (not shown). In ovaries of 50MXC, the number of $\mathrm{AMH}$-positive follicles increased and most follicles showed a more widespread and stronger staining (Fig. 4C and D). The majority of growing follicles, with the exception of some antral follicles (Fig. 4C; arrowhead), had strong, widespread staining in the granulosa cells. Antral follicles stained only in cumulus granulosa cells. In 500MXC ovaries, nearly all follicles other than primordial follicles showed strong $\mathrm{AMH}$ immunostaining (Fig. $4 \mathrm{G}$ and $\mathrm{H}$ ). In 100MXC ovaries, the number of AMH-positive follicles and staining intensity was intermediary to the 50 and $500 \mathrm{MXC}$ (Fig. 4E and F). Higher magnification micrographs revealed stronger immunoreactivity in granulosa cells in 50, 100, and 500MXC ovaries (Fig. 4D, F, and $\mathrm{H}$ insets) as compared with granulosa cells in control ovaries (Fig. 4B inset). In control ovaries, granulosa cells had relatively limited AMH immunoreactivity in follicles beyond the primary follicle stage as compared with MXC-treated ovaries. Negativecontrol ovaries showed no specific immunoreactivity (data not shown).

\section{Effect of MXC on ovarian AMH production in vivo}

To quantify ovarian AMH production, western blot analysis was performed. The results of western blot analysis 

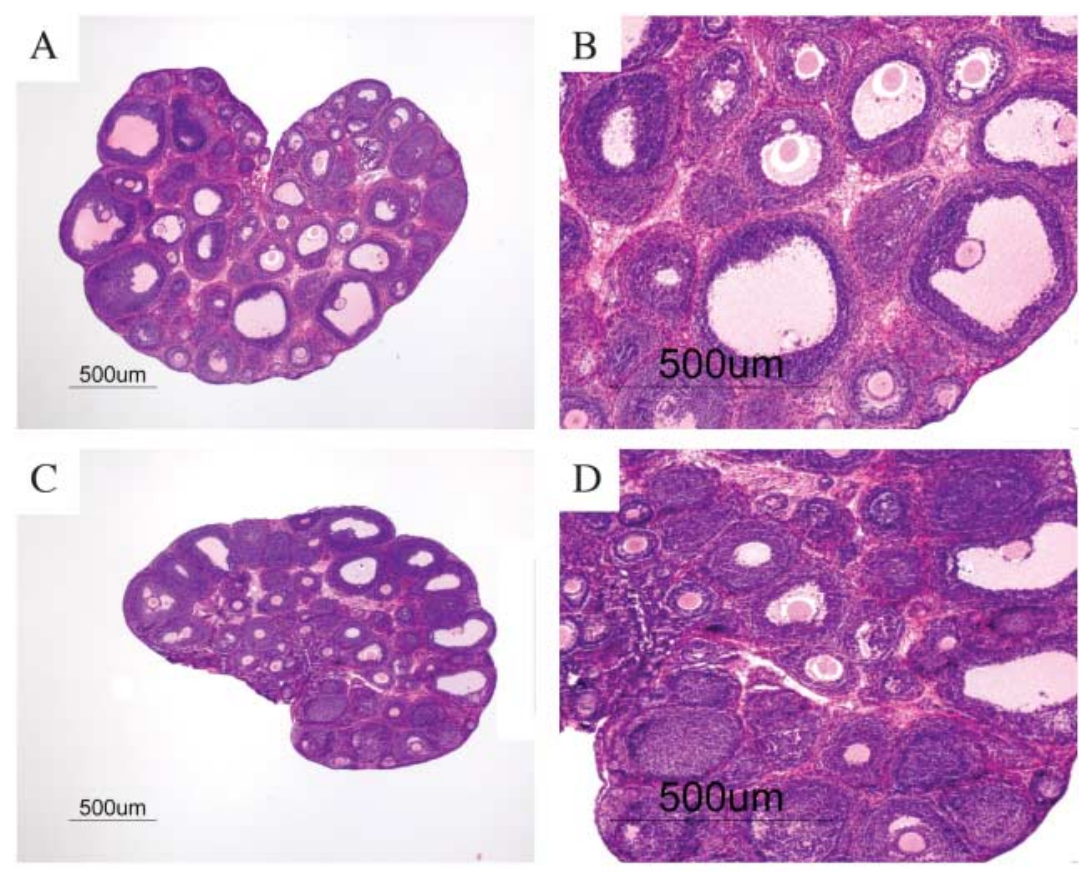

$\mathrm{E}$

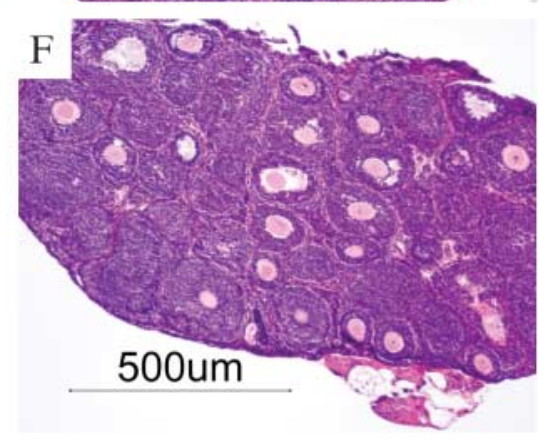

Figure 2 Effect of early postnatal methoxychlor (MXC) exposure on size of and folliculogenesis in the rat ovary. Histology was performed as described in Materials and Methods. (A and B), controls; (C and D), $50 \mathrm{mg} / \mathrm{kg}$ per day MXC; (E and F), $500 \mathrm{mg} / \mathrm{kg}$ per day MXC. Daily MXC injections (s.c.) between P3 and P10 reduced ovarian size in a dose-dependent manner by P20 (C and E) compared with controls (A). In addition, while control ovaries had many small, medium, and large antral follicles (B), MXC treatment caused dose-dependent reduction in the number of medium and large antral follicles in the ovary (D and F). Ovaries treated with $500 \mathrm{mg} / \mathrm{kg}$ per day MXC had no medium or large antral follicles (F). The figures are representative of approximately ten ovaries in each treatment group.

supported our observations in IHC and showed that ovaries of 50,100 , and $500 \mathrm{MXC}$-treated females contained $1 \cdot 6 \pm 0 \cdot 2,1 \cdot 85 \pm 0 \cdot 6$, and $2 \cdot 2 \pm 0 \cdot 5$ times more AMH protein than the control ovaries respectively (Fig. 5A and $\mathrm{B}$ ), the differences being statistically significant $(P<0 \cdot 05)$. The AMH protein production in ovaries of 1 and 10MXC was not significantly different from control ovaries $(P>0 \cdot 05)$. The molecular weight of the $\mathrm{AMH}$ band was approximately $65 \mathrm{kDa}$, which is similar to a previously reported result for western blot analysis of bovine AMH separated under reducing conditions (Wilson et al. 1993).
Effect of HPTE on AMH production in rat granulosa cells in vitro

To assess whether increased ovarian AMH production following MXC treatment in vivo was due to a direct action of MXC or its metabolites in the ovary, we examined the effect of HPTE on granulosa cells as HPTE is more active (e.g. has a higher affinity for estrogen receptor (ER)) than MXC. While $0.5 \mu \mathrm{M}$ HPTE stimulated AMH production, this was not significant $(P>0 \cdot 05)$. However, 1 and $5 \mu \mathrm{M}$ HPTE significantly stimulated AMH production $(P<0 \cdot 05$; Fig. 6$)$. Higher doses of HPTE (10 and $25 \mu \mathrm{M})$ stimulated the AMH production but this was not 
A

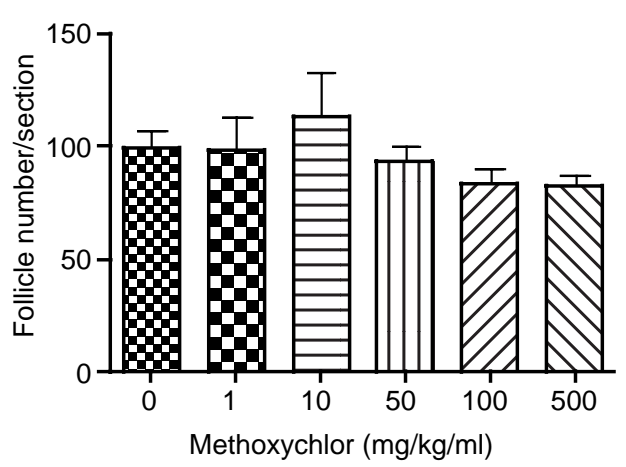

C

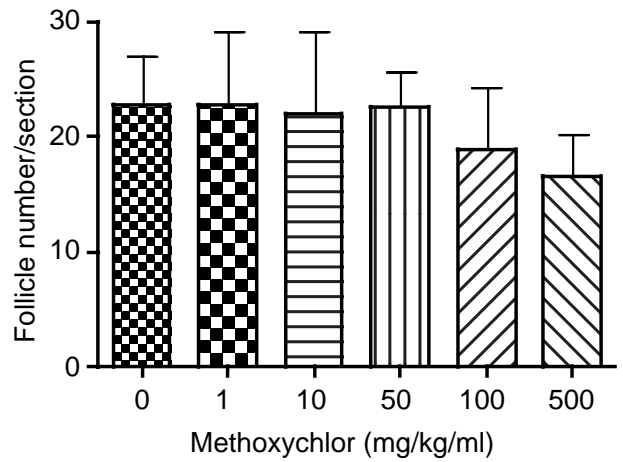

E

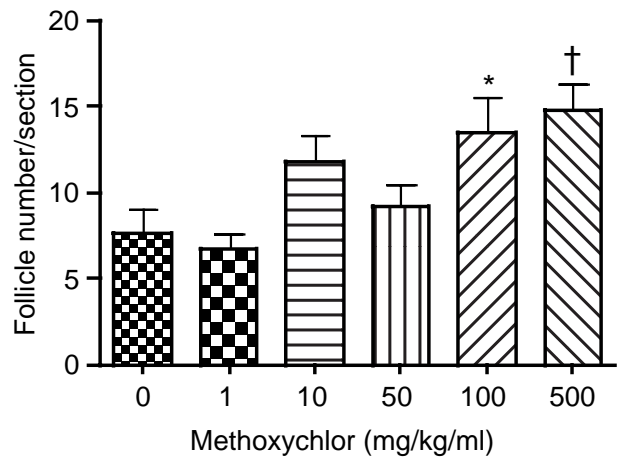

B

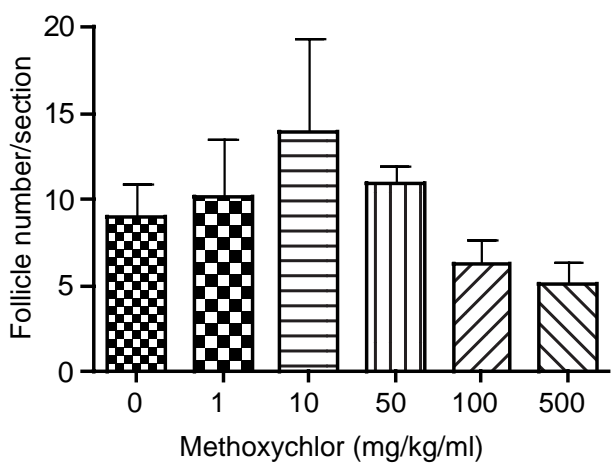

D

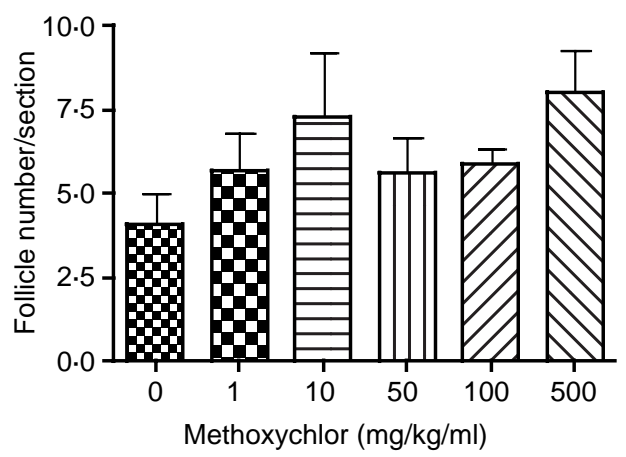

$\mathrm{F}$

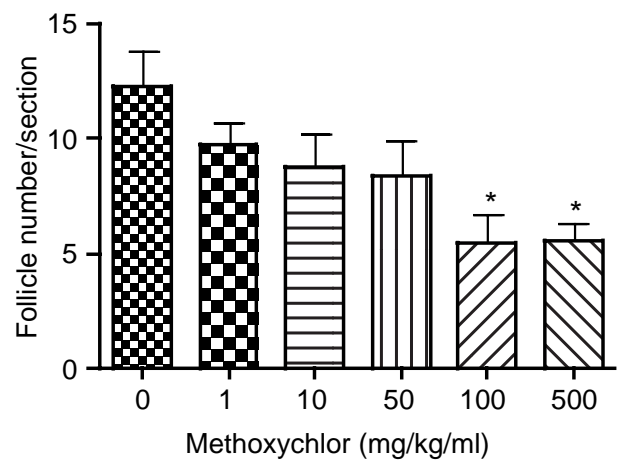

Figure 3 Effect of early postnatal MXC exposure on follicle number and stage in the P20 rat ovary. The rats were injected (s.c.) daily with MXC (1-500 mg/kg per day) between P3 and P10; (A) total follicle numbers were determined; and follicles were assigned into (B) primordial, (C) primary, (D) secondary, (E) pre-antral, or (F) antral stages as described in Materials and Methods. Exposure to 100 and $500 \mathrm{mg} / \mathrm{kg}$ per day MXC decreased antral follicles with a concomitant increase in pre-antral follicle numbers. Total follicle number and the number of primordial, primary, and secondary follicles were not different between the treatment groups. The experiments were repeated two to five times with at least two to three animals in each experimental repeat. The data are representative of $5-11$ ovaries in each treatment group. ${ }^{*} P<0 \cdot 05,{ }^{\dagger} P<0 \cdot 01$.

significant $(P>0 \cdot 05)$, and $25 \mu \mathrm{M}$ HPTE appeared to be toxic to the cells since some of the cells detached (not shown). A similar result was obtained when the experiment was done using granulosa cells harvested from immature rats, primed with $15 \mathrm{IU}$ eCG (s.c.) for $24 \mathrm{~h}$ (data not shown).

\section{Discussion}

The present study shows that neonatal MXC treatment inhibits follicular development and reduces antral follicle numbers in the ovary. In addition, MXC treatment increases the level of AMH protein production in the ovary. This study 

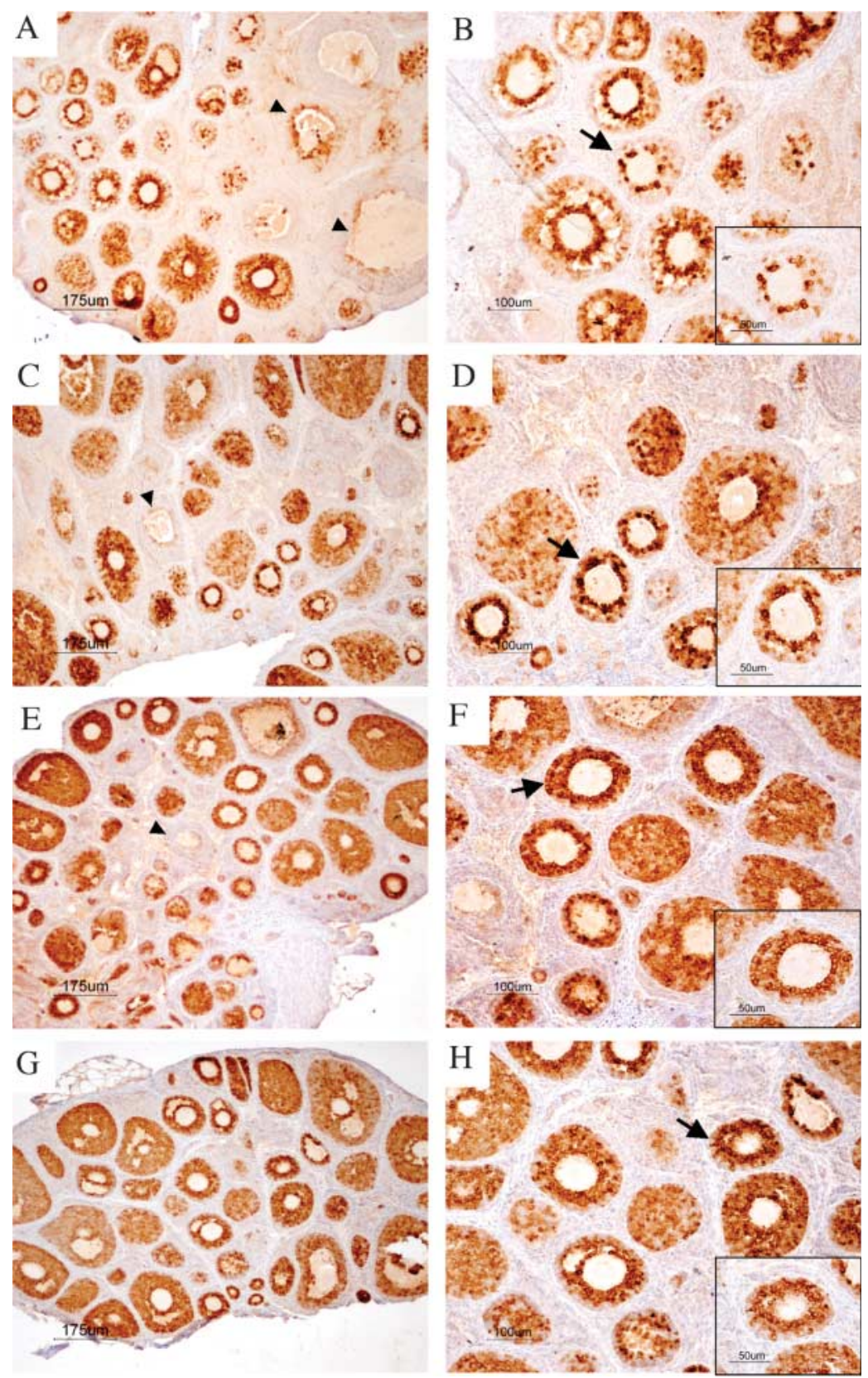

Figure 4 Effect of early postnatal methoxychlor (MXC) exposure on $\mathrm{AMH}$ immunolocalization in the P20 rat ovary. Neonatal female rats were injected (s.c.) daily with $0-500 \mathrm{mg} / \mathrm{kg}$ per day MXC between P3 and P10, and ovaries were collected on P20. AMH immunohistochemistry was performed in the ovaries as described in Materials and Methods. (A and B), Controls; (C and D), $50 \mathrm{mg} / \mathrm{kg}$ per day MXC; (E and F), $100 \mathrm{mg} / \mathrm{kg}$ per day MXC; ( $\mathrm{G}$ and $\mathrm{H}), 500 \mathrm{mg} / \mathrm{kg}$ per day MXC. AMH protein immunoreactivity (brown color) was observed in granulosa cells of growing pre-antral and early antral follicles. In some antral follicles, staining was limited to cumulus granulosa cells or the staining was absent (arrowheads). There were more AMHpositive follicles in MXC-treated ovaries (C, E, and G) as compared with control ovaries (A). $\mathrm{AMH}$ immunostaining intensity in the follicles (i.e. granulosa cells) was stronger in MXCtreated ovaries (D, F, and $\mathrm{H}$ ) as compared with control ovaries (B). A representative follicle (arrows) is shown at a larger magnification (B, D, F, and $\mathrm{H}$; and insets). Negative control sections that were incubated with normal goat IgG or $\mathrm{AMH}$ antibody and excess blocking peptide instead of anti-AMH antibody showed no specific staining (not shown). The figures represent approximately ten ovaries for each treatment group. 
A

MXC

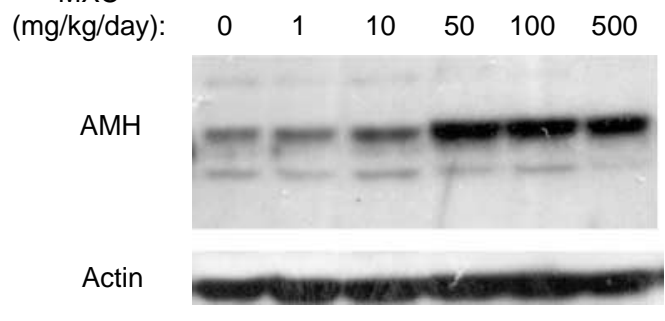

$\mathrm{B}$

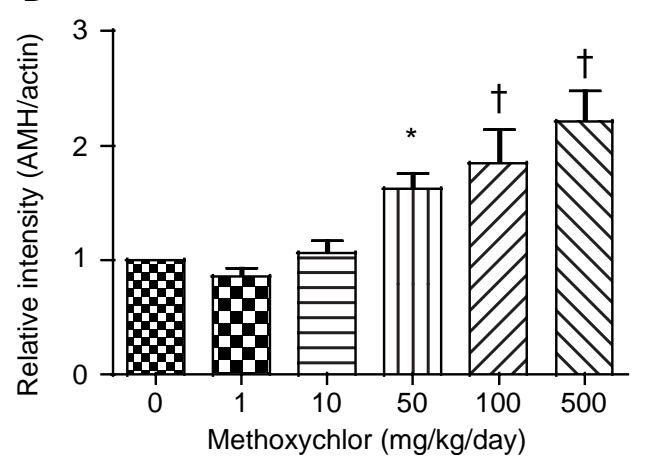

Figure 5 Effect of early postnatal methoxychlor (MXC) exposure on $\mathrm{AMH}$ production in the P20 rat ovary. Neonatal female rats were injected daily between P3 and P10, and ovaries were collected on P20. Western blot analysis was conducted in the ovaries as described in Materials and Methods. (A) Representative western blots of AMH protein. Actin was used as a loading control. (B) Mean and S.E.M. of percent relative intensity of $\mathrm{AMH}$ bands in western blot analysis of three independent experiments from ovaries of MXCtreated rats in comparison with the controls. The control band intensities were adjusted to $1(n=3) .{ }^{*} P<0 \cdot 05,{ }^{\dagger} P<0 \cdot 01$.

shows for the first time that the production of AMH by granulosa cells is increased following HPTE treatment. Overall, these data suggest a local inhibitory effect of MXC directly on the ovary.

MXC treatment causes a reduction in the weight and size of the ovary. In addition, while the ovaries of each MXC treatment had approximately the same total number of follicles, MXC-treated ovaries had a dose-dependent reduction in the number of antral follicles with a concomitant increase in pre-antral stage follicles. This is similar to previous observations that neonatal estrogen treatment causes a reduction in the number of medium and large antral follicles (Ikeda et al. 2001, 2002). A recent in vitro study has shown that estrogen treatment reduces the number of growing pre-antral follicles, suggesting that estrogen inhibits the transition from primordial to primary follicle stage (Kezele \& Skinner 2003). In the present study, MXC-treated ovaries had fewer antral follicles without an apparent reduction in total follicle number. This suggests that the effect of MXC on follicular development can be at any stage, such as the primordialto-primary follicle transition, growth of the primary follicle into a more advanced pre-antral follicle, or recruitment of

\section{A}

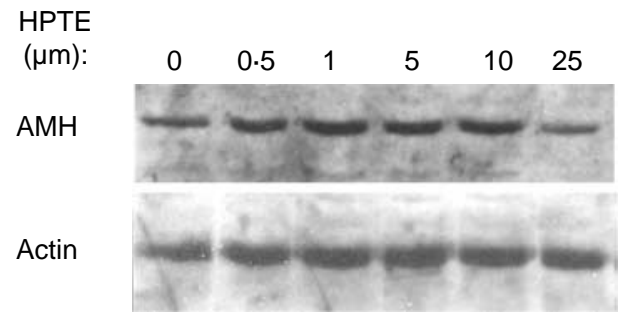

B

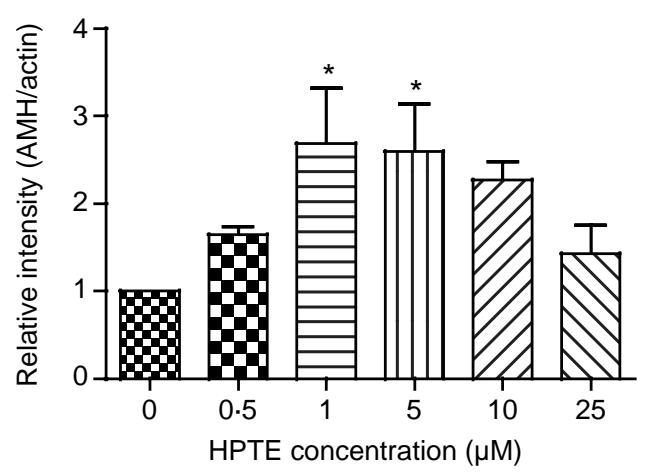

Figure 6 Effect of HPTE on AMH production in rat granulosa cells in vitro. DES-primed immature rat granulosa cells were prepared and treated with HPTE (0-25 $\mu \mathrm{M}$ HPTE), and western blot analysis was conducted using these granulosa cells as described in Materials and Methods. (A) Representative western blots of AMH protein. Actin was used as a loading control. (B) Mean and S.E.M. of percent relative intensity of $\mathrm{AMH}$ bands in western blot analysis of three independent experiments with HPTE-treated granulosa cells in comparison with the control cells. The control band intensities were adjusted to $1(n=3) .{ }^{*} P<0 \cdot 05$.

pre-antral follicles into the antral follicle stage. Based on the follicle numbers, it seems that the inhibition of MXC may be on the latter: the recruitment of pre-antral follicles into the antral follicle stage. Further investigation, such as follicle counts in multiple sections throughout the entire ovary, is needed to confirm this finding.

Due to its estrogenic actions, MXC may provide a negative feedback on the hypothalamus and pituitary and reduce gonadotropin secretion, which can inhibit ovarian folliculogenesis in treated rats. The pituitary hormones are present in pre-pubertal rats. The level of FSH is low at birth and reaches its peak value between $\mathrm{P} 12$ and $\mathrm{P} 15$, after which it returns to the initial low level and stays low until P45 (Ojeda \& Ramirez 1972). Estradiol normally cannot induce a negative feedback on the hypothalamus and pituitary in pre-pubertal rats because of serum $\alpha$-fetoprotein, which binds to serum estradiol and does not allow it to move into tissues, such as the hypothalamus and pituitary (Greenstein 1992). Whether MXC can transfer into these tissues is not known. However, if it does, MXC can potentially act on these tissues because $\operatorname{ER} \alpha$ and $\operatorname{ER} \beta$ are expressed in the hypothalamus and pituitary of neonatal female rats (Nishihara et al. 2000, Ikeda et al. 2003). It has been 
speculated that neonatal MXC exposure leading to cycle irregularities and ovarian pathology in mice is probably due to altered hypothalamic-pituitary function (Eroschenko et al. 1995). However, whether MXC affects the hypothalamus and pituitary is controversial. Injection of MXC $(8-64 \mathrm{mg} / \mathrm{kg}$ per day) in adult mice for 20 days did not change the serum FSH levels or levels of the FSH receptor in the ovary (Borgeest et al. 2004). In addition, feeding MXC (24-1200 ppm) to pregnant rats between embryonic day 15 (E15) and P10 does not affect the size of the sexually dimorphic nucleus of the pre-optic area of the hypothalamus in the female offspring (Masutomi et al. 2003). On the other hand, perinatal (E15-P10) exposure to $\mathrm{MXC}$ has been reported to inhibit serum LH levels in adult female rats (Suzuki et al. 2004). Similarly, treatment with 50 or $100 \mathrm{mg} / \mathrm{kg}$ per day MXC for 1 week before and 6 weeks after birth suppressed FSH levels during estrus (Chapin et al. 1997). In addition, preliminary results from our laboratory suggest that MXC treatment between P3 and P10, suppressed serum FSH levels on P8-P16 (Clayton \& M Uzumcu, unpublished observations). Studies are underway to confirm the preliminary results and to further investigate the exact role of suppressed gonadotropins in the observed inhibition of folliculogenesis in the present study.

In addition to its potential action through the hypothalamicpituitary-ovarian (HPO) axis, MXC and/or its metabolites probably act directly on the ovary. It is possible that $\mathrm{MXC}$ stimulates ovarian AMH production directly, which in turn inhibits folliculogenesis. In the present study, the increased intensity of the AMH immunostaining in individual granulosa cells or follicles in MXC-treated ovaries suggests that MXC acts on the ovary directly. This suggestion was clearly supported by our results which show that HPTE, one of the major metabolites of $\mathrm{MXC}$, significantly stimulated $\mathrm{AMH}$ production in DES-primed rat granulosa cells in vitro. This novel finding is somewhat expected, since MXC has been shown to directly increase antral follicle atresia through several mechanisms, including the Bcl-2 and Bax-mediated pathway (Miller et al. 2005), oxidative stress-mediated pathway (Gupta et al. 2006), and estrogen signaling-mediated pathway (Miller et al. 2006, Tomic et al. 2006) in a follicle culture system. In addition, estrogen and estrogenic compounds, such as MXC and its metabolites, are known to regulate ovarian $\mathrm{AMH}$ gene expression. The AMH promoter has a 13 base pair sequence that differs only by one nucleotide from the estrogen responsive element (ERE) of the vitellogenin gene found 1722 base pairs upstream of the human gene. Co-transfection of ER and ERE inverted repeat as 35 base pair oligonucleotide linked to a reported gene confers estrogen responsiveness on a heterologous reporter (Guerrier et al. 1990). Elevated AMH can inhibit folliculogenesis, since AMH inhibits the primordialto-primary follicle transition (Durlinger et al. 2002) and FSHinduced follicular development (di Clemente et al. 1994) in the ovary. Data presented from the present study as well as studies by others suggest that the inhibition of folliculogenesis that was observed in this study can be due to direct action on the ovaryprobably through increased ovarian AMH production.
In conclusion, this study shows that neonatal exposure to the endocrine disruptor MXC inhibits ovarian follicular development and reduces antral follicle numbers in rats. In addition, MXC, possibly through its major metabolite HPTE, stimulates AMH production in the ovary. Thus, the inhibitory effect of MXC on the ovary may, at least in part, be directly on the ovary and mediated by the local paracrine factor AMH. This study highlights the potential adverse effects of endocrine disruptors on ovarian pathology and female fertility, which can be mediated by local paracrine factors such as $\mathrm{AMH}$.

\section{Acknowledgements}

The authors greatly acknowledge Drs Michael Skinner (Washington State University, Pullman, WA, USA) and Hiroetsu Suzuki (Nippon Veterinary and Animal Science University Tokyo, Japan) for their conceptual inputs. The authors wish to thank Dr Kathy Manger for her assistance in the preparation of the manuscript and Sophie Ogureck and Michael Esmail for their help in caring for the rats. The research is supported by funding from Rutgers University NJAES Hatch Grant and NIEHS grant P30 ES05022-17. There is no conflict of interest that would prejudice the impartiality of this research.

\section{References}

Bedell MA, Brannan CI, Evans EP, Copeland NG, Jenkins NA \& Donovan PJ

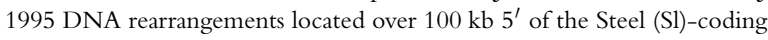
region in Steel-panda and Steel-contrasted mice deregulate Sl expression and cause female sterility by disrupting ovarian follicle development. Genes and Development 9 455-470.

Borgeest C, Symonds D, Mayer LP, Hoyer PB \& Flaws JA 2002 Methoxychlor may cause ovarian follicular atresia and proliferation of the ovarian epithelium in the mouse. Toxicological Sciences 68 473-478.

Borgeest C, Miller KP, Gupta R, Greenfeld C, Hruska KS, Hoyer P \& Flaws JA 2004 Methoxychlor-induced atresia in the mouse involves Bcl-2 family members, but not gonadotropins or estradiol. Biology of Reproduction $\mathbf{7 0}$ 1828-1835.

Chapin RE, Harris MW, Davis BJ, Ward SM, Wilson RE, Mauney MA, Lockhart AC, Smialowicz RJ, Moser VC, Burka LT et al. 1997 The effects of perinatal/juvenile methoxychlor exposure on adult rat nervous, immune, and reproductive system function. Fundamental and Applied Toxicology $\mathbf{4 0}$ 138-157.

di Clemente N, Goxe B, Rémy JJ, Cate RL, Josso N, Vigier B \& Salesse R 1994 Inhibitory effect of AMH upon the expression of aromatase activity and $\mathrm{LH}$ receptors by cultured granulosa cells of rat and porcine immature ovaries. Endocrine 2 553-558.

Cummings AM 1997 Methoxychlor as a model for environmental estrogens. Critical Reviews in Toxicology 27 367-379.

Dong J, Albertini DF, Nishimori K, Kumar TR, Lu N \& Matzuk MM 1996 Growth differentiation factor-9 is required during early ovarian folliculogenesis. Nature 383 531-535.

Durlinger AL, Kramer P, Karels B, de Jong FH, Uilenbroek JT, Grootegoed JA \& Themmen AP 1999 Control of primordial follicle recruitment by antiMullerian hormone in the mouse ovary. Endocrinology 140 5789-5796. 
Durlinger AL, Gruijters MJ, Kramer P, Karels B, Ingraham HA, Nachtigal MW, Uilenbroek JT, Grootegoed JA \& Themmen AP 2002 Anti-Mullerian hormone inhibits initiation of primordial follicle growth in the mouse ovary. Endocrinology 143 1076-1084.

Eroschenko VP, Abuel-Atta AA \& Grober MS 1995 Neonatal exposures to technical methoxychlor alters ovaries in adult mice. Reproductive Toxicology 9 379-387.

Gaido KW, Maness SC, McDonnell DP, Dehal SS, Kupfer D \& Safe S 2000 Interaction of methoxychlor and related compounds with estrogen receptor alpha and beta, and androgen receptor: structure-activity studies. Molecular Pharmacology $\mathbf{5 8} 852-858$.

Greenstein BD 1992 Effects of rat alpha-fetoprotein administration on estradiol free fraction, the onset of puberty, and neural and uterine nuclear estrogen receptors. Endocrinology 130 3184-3190.

Guerrier D, Boussin L, Mader S, Josso N, Kahn A \& Picard JY 1990 Expression of the gene for anti-Mullerian hormone. Journal of Reproduction and Fertility 88 695-706.

Gunderson EL 1988 FDA Total Diet Study, April 1982-April 1984, dietary intakes of pesticides, selected elements, and other chemicals. Journal - Association of Official Analytical Chemists 71 1200-1209.

Gupta RK, Miller KP, Babus JK \& Flaws JA 2006 Methoxychlor inhibits growth and induces atresia of antral follicles through an oxidative stress pathway. Toxicological Sciences 93 382-389.

Hall DL, Payne LA, Putnam JM \& Huet-Hudson YM 1997 Effect of methoxychlor on implantation and embryo development in the mouse. Reproductive Toxicology 11 703-708.

Hirshfield AN 1991 Development of follicles in the mammalian ovary. International Review of Cytology 124 43-101.

Huang EJ, Manova K, Packer AI, Sanchez S, Bachvarova RF \& Besmer P 1993 The murine steel panda mutation affects kit ligand expression and growth of early ovarian follicles. Developmental Biology 157 100-109.

Ikeda Y, Nagai A, Ikeda MA \& Hayashi S 2001 Neonatal estrogen exposure inhibits steroidogenesis in the developing rat ovary. Developmental Dynamics 221 443-453.

Ikeda Y, Nagai A, Ikeda MA \& Hayashi S 2002 Increased expression of Mullerian-inhibiting substance correlates with inhibition of follicular growth in the developing ovary of rats treated with E2 benzoate. Endocrinology 143 304-312.

Ikeda Y, Nagai A, Ikeda MA \& Hayashi S 2003 Sexually dimorphic and estrogen-dependent expression of estrogen receptor beta in the ventromedial hypothalamus during rat postnatal development. Endocrinology 144 5098-5104.

Kezele P \& Skinner MK 2003 Regulation of ovarian primordial follicle assembly and development by estrogen and progesterone: endocrine model of follicle assembly. Endocrinology 144 3329-3337.

Maness SC, McDonnell DP \& Gaido KW 1998 Inhibition of androgen receptor-dependent transcriptional activity by DDT isomers and methoxychlor in HepG2 human hepatoma cells. Toxicology and Applied Pharmacology 151 135-142.

Masutomi N, Shibutani M, Takagi H, Uneyama C, Takahashi N \& Hirose M 2003 Impact of dietary exposure to methoxychlor, genistein, or diisononyl phthalate during the perinatal period on the development of the rat endocrine/reproductive systems in later life. Toxicology 192 149-170.
Miller KP, Gupta RK, Greenfeld CR, Babus JK \& Flaws JA 2005 Methoxychlor directly affects ovarian antral follicle growth and atresia through Bcl-2- and bax-mediated pathways. Toxicological Sciences $\mathbf{8 8}$ 213-221.

Miller KP, Gupta RK \& Flaws JA 2006 Methoxychlor metabolites may cause ovarian toxicity through estrogen regulated pathways. Toxicological Sciences 93 180-188.

Nishihara E, Nagayama Y, Inoue S, Hiroi H, Muramatsu M, Yamashita S \& Koji T 2000 Ontogenetic changes in the expression of estrogen receptor alpha and beta in rat pituitary gland detected by immunohistochemistry. Endocrinology 141 615-620.

Ojeda SR \& Ramirez VD 1972 Plasma level of LH and FSH in maturing rats: response to hemigonadectomy. Endocrinology 90 466-472.

Oktay K, Schenken RS \& Nelson JF 1995 Proliferating cell nuclear antigen marks the initiation of follicular growth in the rat. Biology of Reproduction 53 295-301.

Parrott JA \& Skinner MK 1999 Kit-ligand/stem cell factor induces primordial follicle development and initiates folliculogenesis. Endocrinology 140 4262-4271.

Pepling ME \& Spradling AC 2001 Mouse ovarian germ cell cysts undergo programmed breakdown to form primordial follicles. Developmental Biology 234 339-351.

Suzuki M, Lee HC, Chiba S, Yonezawa T \& Nishihara M 2004 Effects of methoxychlor exposure during perinatal period on reproductive function after maturation in rats. Journal of Reproduction and Development 50 455-461.

Tomic D, Frech MS, Babus JK, Gupta RK, Furth PA, Koos RD \& Flaws JA 2006 Methoxychlor induces atresia of antral follicles in ER \{alpha\} overexpressing mice. Toxicological Sciences 93 196-204.

Uzumcu M \& Lin YC 1994 Characterization of the stimulatory actions of thymic factor(s) on basal and gonadotropin-induced steroidogenesis in cultured rat granulosa cells. Molecular and Cellular Endocrinology 105 209-216.

Uzumcu M, Homsi MF, Ball DK, Coskun S, Jaroudi K, Hollanders JM \& Brigstock DR 2000 Localization of connective tissue growth factor in human uterine tissues. Molecular Human Reproduction 6 1093-1098.

Uzumcu M, Westfall SD, Dirks KA \& Skinner MK 2002 Embryonic testis cord formation and mesonephric cell migration requires the phosphotidylinositol 3-kinase signaling pathway. Biology of Reproduction 67 1927-1935.

Wilson CA, di Clemente N, Ehrenfels C, Pepinsky RB, Josso N, Vigier B \& Cate RL 1993 Mullerian inhibiting substance requires its N-terminal domain for maintenance of biological activity, a novel finding within the transforming growth factor-beta superfamily. Molecular Endocrinology 7 247-257.

Zachow R \& Uzumcu M 2006 The methoxychlor metabolite, 2,2-bis(p-hydroxyphenyl)-1,1,1-trichloroethane, inhibits steroidogenesis in rat ovarian granulosa cells in vitro. Reproductive Toxicology In press.

Received in final form 31 August 2006

Accepted 8 September 2006

Made available online as an Accepted Preprint 27 September 2006 